\title{
Full-Wave Analysis of Microstrip Antennas in Three-Layered Spherical Media
}

\author{
Tao Yu and Chengyou Yin \\ Department of Radar Engineering, Hefei Electronic Engineering Institute, 460 Huangshan Road, Hefei, Anhui 230037, China \\ Correspondence should be addressed to Chengyou Yin; cyouyin@sina.com
}

Received 31 December 2012; Revised 29 June 2013; Accepted 16 July 2013

Academic Editor: Mugen Peng

Copyright ( $) 2013$ T. Yu and C. Yin. This is an open access article distributed under the Creative Commons Attribution License, which permits unrestricted use, distribution, and reproduction in any medium, provided the original work is properly cited.

\begin{abstract}
A model of three-layered spherical microstrip antenna has been analyzed based on Rao-Wilton-Glisson (RWG) triangular basis functions using mixed potential integral equation (MPIE). Firstly, the model of antenna and the dyadic Green's function in spherical microstrip antennas are given at the beginning of this paper. Then, due to the infinite series convergence problem, asymptotic extraction approach is presented to accelerate the Green's functions convergence speed when source and field points are located in the same layer and different layers. The convergence speed can be accelerated observably by using this method. Finally, in order to simplify impedance matrix elements calculation at the junction of the probe and patch, a novel division fashion of pair of triangles is adopted in this paper. The input impedance result obtained shows the validity and effectiveness of the analysis method comparing with published data.
\end{abstract}

\section{Introduction}

Nowadays, conformal microstrip antennas have been the subject of several research works, where cylindrical, spherical, and conical microstrip antennas have been studied [13]. Among the studies, the spherical microstrip antenna is found to be helpful in improving width of beam problem involved with the planar arrays at low elevation [4]. The spherical microstrip antenna is the emphasis of research in this paper.

Several methods have been used to analyze the characteristics of spherical microstrip antenna. The analysis methods include mainly generalized transmission line model (GTLM) analysis, cavity model theory, and full-wave method. The process of the GTLM analysis [5] and cavity model theory [6] is simple; however, the result obtained is accurate only when the substrate thickness is much smaller than wavelength and the radius of the sphere $(h / \lambda<0.01)$. On the contrary, the full-wave method [7] is complicated with accurate and rigorous calculated result, which is adopted widely without the substrate thickness limitation.

In aforementioned study methods, the method of moments based on full-wave solution has been adopted widely in analysis. In general, Green's functions should be considered in the calculated process. The dyadic Green's functions for simple spherical geometry have been presented earlier in [8]. A general expression of dyadic Green's functions in a spherically arbitrary multilayered medium is introduced in $[9,10]$ by applying the method of scattering superposition, where the functions have been expressed in the form of an infinite series of spherical eigenmodes. Another type of Green's function has been given by using the GLDMULT algorithm that calculates spectral-domain Green's function of multilayer spherical, circular, cylindrical, and planar structures, and more details about GLDMULT algorithm can be found in [11]. Based on the GLDMULT algorithm, spherical stacked-patch arrays with emphasis on the physical interpretation of mutual coupling are analyzed in [12]. In [13], a rigorous MoM model that can be used to analyze spherical antennas of any configuration and substrate thickness is presented, and the dyadic Green's functions are decomposed into fast and slow convergent parts, with the slower part incorporated into a single scalar potential expression. Other jobs have been done to accelerate the infinite summation convergence $[14,15]$, with the source and field points located in the same layer and different layers. A probe-fed circularly polarized loop antenna printed on a layered dielectric sphere has been studied in [16]. 


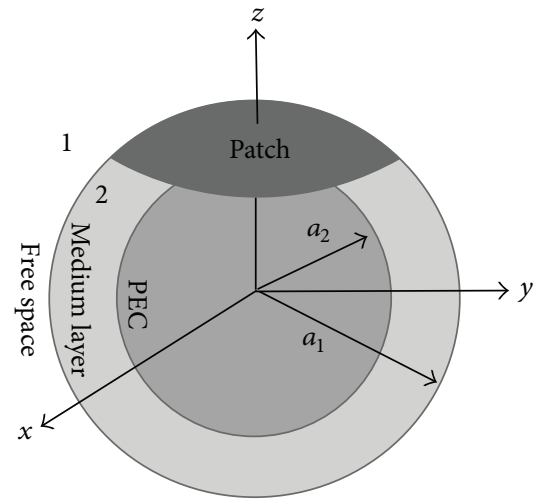

FIGURE 1: The microstrip antenna in a layered spherical media.

In this paper, the model of three-layered spherical microstrip antenna is established, where the problem about substrate thickness and feeding probe is considered. The method of moment based on Rao-Wilton-Glisson triangular basis functions and the dyadic Green's functions in [9] is used in the model using mixed potential integral equation (MPIE). Asymptotic extraction approach is presented to accelerate the Green's functions convergence speed, and a novel division fashion of pair of triangles is adopted to simplify impedance matrix at the junction of the probe and patch. The input impedance of microstrip antennas in spherically layered media is calculated, and calculated results of the input impedance are in good agreement with published data, which shows the validity and effectiveness of the analysis method.

\section{Theory and Formulation}

The model of three-layered spherical microstrip antenna is presented in Figure 1. The patch is located in the free space while the probe is located in the medium layer. A conducting spherical core can be modeled as the inner-most layer.

2.1. Expressions of Dyadic Green's Functions. The electromagnetic dyadic Green's functions in the layered media have been deduced in detail in [9]. The dyadic Green's function can be considered as the sum of an unbounded dyadic Green's function $\overline{\mathbf{G}}_{o e}\left(\mathbf{r}, \mathbf{r}^{\prime}\right)$ and a scattering dyadic Green's function $\overline{\mathbf{G}}_{e s}^{(f s)}\left(\mathbf{r}, \mathbf{r}^{\prime}\right)$, and the unbounded dyadic Green's function can be expressed as

$$
\overline{\mathbf{G}}_{o e}\left(\mathbf{r}, \mathbf{r}^{\prime}\right)=\left(\overline{\mathbf{I}}+\frac{1}{k_{s}^{2}} \nabla \nabla^{\prime}\right) \frac{e^{-j k_{s}\left|\mathbf{r}-\mathbf{r}^{\prime}\right|}}{4 \pi\left|\mathbf{r}-\mathbf{r}^{\prime}\right|},
$$

which can be employed only when the source and field points are located in the same layer [13]. In this paper, according to research requirement, four types of $\overline{\mathbf{G}}_{e s}^{(f s)}\left(\mathbf{r}, \mathbf{r}^{\prime}\right)$ should be considered ( $f$ denotes the layer number of field point's position and $s$ denotes the layer number of source point's position as shown in Figure 1), including $\overline{\mathbf{G}}_{e s}^{(11)}\left(\mathbf{r}, \mathbf{r}^{\prime}\right)$, $\overline{\mathbf{G}}_{e s}^{(21)}\left(\mathbf{r}, \mathbf{r}^{\prime}\right), \overline{\mathbf{G}}_{e s}^{(12)}\left(\mathbf{r}, \mathbf{r}^{\prime}\right)$, and $\overline{\mathbf{G}}_{e s}^{(22)}\left(\mathbf{r}, \mathbf{r}^{\prime}\right)$. To save the space, the expression of all types of Green's functions will not be given here. Only take $\overline{\mathbf{G}}_{e s}^{(11)}\left(\mathbf{r}, \mathbf{r}^{\prime}\right)$ for example:

$$
\begin{gathered}
\overline{\mathbf{G}}_{e s}^{(11)}\left(\mathbf{r}, \mathbf{r}^{\prime}\right)=\frac{-j k_{1}}{4 \pi} \sum_{n=1}^{\infty} \sum_{m=0}^{n}\left(2-\delta_{m}^{0}\right) \frac{2 n+1}{n(n+1)}, \\
\frac{(n-m) !}{(n+m) !} \times\left[\begin{array}{c}
\mathbf{M}^{(2) e}{ }_{o m n}\left(k_{1}\right) B_{M}^{11} \mathbf{M}^{(2) e}{ }_{o m n}\left(k_{1}\right) \\
+\mathbf{N}_{o m n}^{(2) e}\left(k_{1}\right) B_{N}^{11} \mathbf{N}_{o m n}^{(2) e}\left(k_{1}\right)
\end{array}\right],
\end{gathered}
$$

where vector eigenfunctions $\mathbf{M}^{(2)}, \mathbf{M}^{\prime(2)}, \mathbf{N}^{(2)}$, and $\mathbf{N}^{\prime(2)}$ can be found in [9]. In this case, (2) represents Green's function that both the source and field points are located in the free space, and the coefficients $B_{M}^{11}, B_{N}^{11}$ are derived and presented by

$$
B_{M, N}^{11}=-\frac{R_{F 1}^{H, V} \cdot T_{P 1}^{H, V}+T_{F 1}^{H, V} \cdot R_{F 2}^{H, V}}{T_{P 1}^{H, V}+R_{F 2}^{H, V} R_{P 1}^{H, V} T_{F 1}^{H, V}},
$$

where the equivalent reflection and transmission coefficients $R_{(P, F) f}^{H, V}, T_{(P, T) f}^{H, V}$ can be calculated by [9] and cannot be given here. The scattering dyadic Green's function $\overline{\mathbf{G}}_{e s}^{(11)}\left(\mathbf{r}, \mathbf{r}^{\prime}\right)$ contains nine components, which can be obtained through the substitution of general expressions of vector eigenfunction for (2). Take $\hat{\boldsymbol{\theta}} \boldsymbol{\boldsymbol { \theta }}$ component, for example,

$$
G_{\theta \theta}^{11}=\frac{-j k_{1}}{4 \pi} \sum_{n=1}^{\infty} \frac{2 n+1}{n(n+1)}\left\{\begin{array}{c}
B_{M}^{11} h_{n}^{(2)}\left(k_{1} r\right) h_{n}^{(2)}\left(k_{1} r^{\prime}\right) \alpha \\
+B_{N}^{11} \frac{1}{k_{1}^{2} r r^{\prime}} \frac{d\left[r h_{n}^{(2)}\left(k_{1} r\right)\right]}{d r} \\
\times \frac{d\left[r^{\prime} h_{n}^{(2)}\left(k_{1} r^{\prime}\right)\right]}{d r^{\prime}} \beta
\end{array}\right\},
$$

where

$$
\begin{gathered}
\alpha=-\sin ^{2}\left(\phi-\phi^{\prime}\right) \sin \theta \sin \theta^{\prime} \frac{P_{n}^{2}(\cos \gamma)}{(\sin \gamma)^{2}} \\
+\cos \left(\phi-\phi^{\prime}\right) \frac{\partial P_{n}(\cos \gamma)}{\partial \cos \gamma} \\
\beta=\frac{P_{n}^{2}(\cos \gamma)}{(\sin \gamma)^{2}}\left(-\cos \theta \sin \theta^{\prime}+\sin \theta \cos \theta^{\prime} \cos \left(\phi-\phi^{\prime}\right)\right) \\
\times\left(-\sin \theta \cos \theta^{\prime}+\cos \theta \sin \theta^{\prime} \cos \left(\phi-\phi^{\prime}\right)\right) \\
+\frac{\partial P_{n}(\cos \gamma)}{\partial \cos \gamma} \\
\times\left(\sin \theta \sin \theta^{\prime}+\cos \theta \cos \theta^{\prime} \cos \left(\phi-\phi^{\prime}\right)\right), \\
\cos \gamma=\cos \theta \cos \theta^{\prime}+\sin \theta \sin \theta^{\prime} \cos \left(\varphi-\varphi^{\prime}\right),
\end{gathered}
$$


$h_{n}^{(2)}\left(k_{1} r\right)$ is the spherical Hankel function of the second type, $P_{n}(\cos \gamma)$ is the Legendre function of degree $n$, and $P_{n}^{2}(\cos \gamma)$ is the associated Legendre function of the first kind with the order 2 and degree $n$.

Each component of the scattering dyadic Green's functions can be expressed as an infinite summation of spherical harmonic and be constitutive of two components. Take (4) for example; the first part represents the TE modes, and the second one represents TM modes. Proper truncation of the summation is essential for an infinite summation. The summation of the TE modes converges much faster than that of the corresponding TM modes according to the asymptotic spherical Bessel and Hankel functions, and in order to improve the computation efficiency, TM modes of nine components are incorporated into a unified potential $G_{\varphi}$ [13].

The electric field can be expressed using the scattering dyadic Green's functions

$$
\mathbf{E}(\mathbf{r})=-j \omega \mu_{f} \iint_{s} \overline{\mathbf{G}}_{e s}^{f s}\left(\mathbf{r}, \mathbf{r}^{\prime}\right) \cdot \mathbf{J}_{s}\left(\mathbf{r}^{\prime}\right) d s^{\prime} .
$$

Equation (6) also can be expressed as

$$
\begin{aligned}
\mathbf{E}(\mathbf{r})=-j \omega \mu_{f} \iint_{s}\left(\overline{\mathbf{G}}_{e s}^{\mathrm{TE}}\left(\mathbf{r}, \mathbf{r}^{\prime}\right)\right. & \\
& \left.+\overline{\mathbf{G}}_{e s}^{\mathrm{TM}}\left(\mathbf{r}, \mathbf{r}^{\prime}\right)\right) \cdot \mathbf{J}_{s}\left(\mathbf{r}^{\prime}\right) d s^{\prime}, \\
\mathbf{E} & =-j \omega \mathbf{A}-\nabla \phi,
\end{aligned}
$$

where

$$
\begin{aligned}
\mathbf{A} & =\mu_{f} \iint_{s^{\prime}} \overline{\mathbf{G}}_{A} \cdot \mathbf{J}_{s}\left(r^{\prime}\right) d s^{\prime}, \\
\phi & =\iint_{s^{\prime}} \nabla^{\prime} G_{\varphi} \cdot \mathbf{J}_{s}\left(r^{\prime}\right) d s^{\prime} .
\end{aligned}
$$

We can regard the magnetic vector potential $\mathbf{A}$ and the potential scalar $\phi$ as TM modes contributions and TE modes contributions, respectively. Then

$$
\begin{gathered}
\overline{\mathbf{G}}_{A}=\overline{\mathbf{G}}_{e s}^{\mathrm{TE}}, \\
\nabla \phi=j \omega \mu_{f} \iint_{s} \overline{\mathbf{G}}_{e s}^{\mathrm{TM}}\left(\mathbf{r}, \mathbf{r}^{\prime}\right) \cdot \mathbf{J}_{s}\left(\mathbf{r}^{\prime}\right) d s^{\prime},
\end{gathered}
$$

where $\overline{\mathbf{G}}_{A}, G_{\varphi}$ can be found in [13].

2.2. Asymptotic Dyadic Green's Functions. In this paper, asymptotic extraction approach has been adopted which can accelerate the infinite summation convergence [14]. This can be completed by using the large order spherical Bessel and
Hankel functions principal asymptotic expressions:

$$
\begin{aligned}
& j_{n}(k r) \approx \sqrt{\frac{1}{2 k r(2 n+1)}}\left(\frac{e k r}{2 n+1}\right)^{n+(1 / 2)} \quad n \longrightarrow \infty, \\
& h_{n}^{(2)}(k r) \approx j \sqrt{\frac{2}{k r(2 n+1)}}\left(\frac{e k r}{2 n+1}\right)^{-n-(1 / 2)} \quad n \longrightarrow \infty, \\
& \frac{d\left[r j_{n}(k r)\right]}{d r} \approx n j_{n}(k r) \quad n \longrightarrow \infty, \\
& \frac{d\left[r h_{n}^{(2)}(k r)\right]}{d r} \approx-n h_{n}^{(2)}(k r) \quad n \longrightarrow \infty .
\end{aligned}
$$

When the source and field points are located in the free space (the first layer), the Green's function coefficients are shown as

$$
\begin{gathered}
\left.B_{M}^{11}\right|_{a} \approx 0, \\
\left.B_{N}^{11}\right|_{a}=-\frac{\varepsilon_{1}-\varepsilon_{2}}{\varepsilon_{1}+\varepsilon_{2}} \frac{j_{n}\left(k_{1} a_{1}\right)}{h_{n}^{(2)}\left(k_{1} a_{1}\right)}-\frac{\varepsilon_{2}-\varepsilon_{3}}{\varepsilon_{2}+\varepsilon_{3}} \frac{j_{n}\left(k_{1} a_{2}\right)}{h_{n}^{(2)}\left(k_{1} a_{2}\right)},
\end{gathered}
$$

where the subscript $a$ denotes an asymptotic expression. It can be seen that TE modes coefficients asymptote to zero when $n$ is large, while TM modes coefficients depend on the wave reflections at the dielectric. The asymptotic dyadic Green's function can be presented, and take $\widehat{\boldsymbol{\theta}} \hat{\boldsymbol{\theta}}$ component for example:

$\left.G_{\theta \theta}^{11}\right|_{a}=\frac{-j k_{1}}{4 \pi}$

$$
\times \sum_{n=1}^{\infty} \frac{2 n+1}{n(n+1)}\left\{\begin{array}{c}
B_{N}^{11} \frac{d\left[r h_{n}^{(2)}\left(k_{1} r\right)\right]}{d r} \frac{d\left[r^{\prime} h_{n}^{(2)}\left(k_{1} r^{\prime}\right)\right]}{d r^{\prime}} \\
\times \frac{1}{k_{1}^{2} r r^{\prime}} \frac{\partial^{2} P_{n}(\cos \gamma)}{\partial \theta \partial \theta^{\prime}}
\end{array}\right\} ;
$$

using (10d), (12) can be written as

$$
\begin{aligned}
\left.G_{\theta \theta}^{11}\right|_{a}= & \frac{-j}{4 \pi k_{1}} \\
& \times \sum_{n=1}^{\infty}(2 n+1)\left\{\begin{array}{c}
{\left[\left.B_{N}^{11}\right|_{a} h_{n}^{(2)}\left(k_{1} r\right) h_{n}^{(2)}\left(k_{1} r^{\prime}\right)\right]} \\
\times \frac{1}{r r^{\prime}} \frac{\partial^{2} P_{n}(\cos \gamma)}{\partial \theta \partial \theta^{\prime}}
\end{array}\right\},
\end{aligned}
$$


where one assumes $(n+1) \approx n$ for large $n$. The other asymptotic $\overline{\mathbf{G}}_{e s}^{(11)}\left(\mathbf{r}, \mathbf{r}^{\prime}\right)$ components can be derived in the same way and then incorporated into a unified expression:

$$
\begin{aligned}
\left.\overline{\mathbf{G}}_{e s}^{11}\right|_{a}= & \nabla \nabla^{\prime} G_{1}=\frac{j}{4 \pi k_{1}} \nabla \nabla^{\prime} \sum_{n=0}^{\infty}(2 n+1) P_{n}(\cos \gamma) \\
& \times\left\{\left(-\frac{\varepsilon_{1}-\varepsilon_{2}}{\varepsilon_{1}+\varepsilon_{2}}\right) \frac{a_{1}}{r} \times j_{n}\left(k_{1} \frac{a_{1}^{2}}{r}\right) h_{n}^{(2)}\left(k_{1} r^{\prime}\right)\right. \\
& \left.-\left(\frac{\varepsilon_{2}-\varepsilon_{3}}{\varepsilon_{2}+\varepsilon_{3}}\right) \frac{a_{2}}{r} \times h_{n}^{(2)}\left(k_{1} \frac{a_{2}^{2}}{r}\right) j_{n}\left(k_{1} r^{\prime}\right)\right\} ;
\end{aligned}
$$

with the aid of the addition theorem of the spherical Hankel function

$$
\frac{e^{-j k\left|\mathbf{r}-\mathbf{r}^{\prime}\right|}}{4 \pi\left|\mathbf{r}-\mathbf{r}^{\prime}\right|}=\frac{-j k}{4 \pi} \sum_{n=0}^{\infty}(2 n+1) h_{n}^{(2)}\left(k r^{\prime}\right) j_{n}(k r) P_{n}(\cos \gamma),
$$

equation (14) can be reduced to

$$
\begin{aligned}
\left.\overline{\mathbf{G}}_{e s}^{11}\right|_{a}= & \nabla \nabla^{\prime} G_{2}=\frac{j}{4 \pi k_{1}^{2}} \nabla \nabla^{\prime} \\
& \times\left(\left(-\frac{\varepsilon_{1}-\varepsilon_{2}}{\varepsilon_{1}+\varepsilon_{2}}\right) \frac{a_{1}}{r} \frac{e^{-j k_{1} R_{1}}}{R_{1}}+\left(-\frac{\varepsilon_{2}-\varepsilon_{3}}{\varepsilon_{2}+\varepsilon_{3}}\right) \frac{a_{2}}{r} \frac{e^{-j k_{1} R_{2}}}{R_{2}}\right),
\end{aligned}
$$

where $R_{1}=\sqrt{r^{\prime 2}+\left(a_{1}^{2} / r\right)^{2}-2 r^{\prime}\left(a_{1}^{2} / r\right) \cos \gamma}, R_{2}=$ $\sqrt{r^{\prime 2}+\left(a_{2}^{2} / r\right)^{2}-2 r^{\prime}\left(a_{2}^{2} / r\right) \cos \gamma}$, then $\overline{\mathbf{G}}_{e s}^{(11)}\left(\mathbf{r}, \mathbf{r}^{\prime}\right)$ can be expressed as

$$
\overline{\mathbf{G}}_{e s}^{11}=\left(\overline{\mathbf{G}}_{e s}^{11}-\nabla \nabla^{\prime} G_{1}\right)+\nabla \nabla^{\prime} G_{2},
$$

and $\nabla \nabla^{\prime} G_{1}, \nabla \nabla^{\prime} G_{2}$ are given by (14), (16), respectively. The component in (17) right hand bracket is the infinite summation of spherical harmonic; however the Green's functions convergence speed is accelerated. The other component is a close-form representation and can be treated as similar as that of unbounded dyadic Green's function (1). Asymptotic dyadic Green's functions of $\overline{\mathbf{G}}_{e s}^{(21)}\left(\mathbf{r}, \mathbf{r}^{\prime}\right), \overline{\mathbf{G}}_{e s}^{(12)}\left(\mathbf{r}, \mathbf{r}^{\prime}\right)$, and $\overline{\mathbf{G}}_{e s}^{(22)}\left(\mathbf{r}, \mathbf{r}^{\prime}\right)$ can be obtained in the same way, which are given in the Appendix.

2.3. MoM Solution. The method of moments based on RaoWilton-Gilson triangular basis function is adopted in the model using the mixed potential integral equation (MPIE), where the antenna surface and probe are divided into a mesh of triangular patches as shown in Figure 2, and the probe is modeled by strip. A problem that needs special attention is the connection of the feeding probe to the patch. A possible solution [13] is introduced by the double use of the edge shared by the probe and the patch, where the common triangle is located in the probe as shown in Figure 3, which is

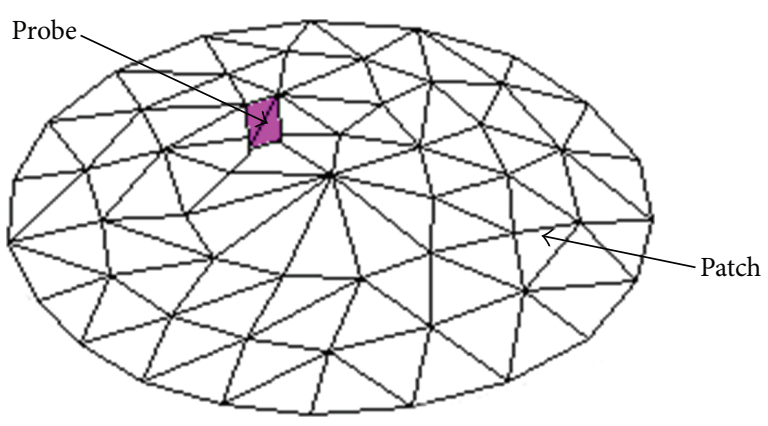

FIGURE 2: Mesh of triangular patches.
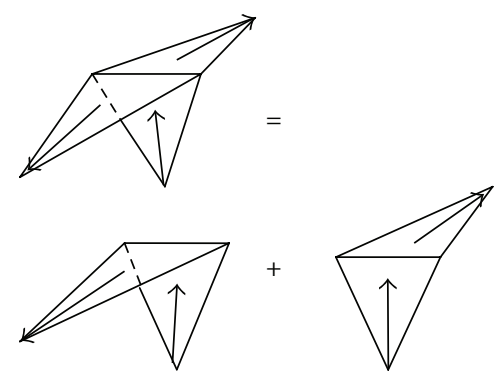

FIGURE 3: Division fashion of pair of triangles in [13].

efficient in [13] because the probe and the patch are located in the same layer, where only a type of dyadic Green's function $\overline{\mathbf{G}}_{e s}^{(22)}\left(\mathbf{r}, \mathbf{r}^{\prime}\right)$ is used in the model.

In this paper, the analysis process is complicated if the aforementioned division fashion is adopted since there are two pairs of RWG basis functions at the junction. A novel division fashion of pair of triangles is adopted to simplify impedance matrix where the common triangle is located in the patch as shown in Figure 4, and only a pair of RWG basis function positions at the junction.

The impedance matrix elements can be calculated based on MoM using the mixed potential integral equation (MPIE):

$$
Z_{m n}=\left\langle\left(j \omega \mathbf{A}_{n}+\nabla \phi_{n}\right), \mathbf{f}_{m}\right\rangle,
$$

where $\mathbf{f}_{m}$ is testing basis function, and

$$
\left\langle\nabla \phi, \mathbf{f}_{m}\right\rangle=\int_{S} \nabla \cdot\left(\phi \mathbf{f}_{m}\right) d S-\int_{s} \phi \nabla \cdot \mathbf{f}_{m} d S,
$$

using Gauss theorem

$$
\int_{s} \nabla \cdot\left(\phi \mathbf{f}_{m}\right) d S=\oint_{l} \phi \mathbf{f}_{m} \cdot \widehat{\mathbf{n}} d l,
$$

where $l$ are three edges around the triangle and $\widehat{\mathbf{n}}$ is normal unit vector. The term $\mathbf{f}_{m} \cdot \widehat{\mathbf{n}}$ equals zero on the boundary edge. To the common edge, (20) equals zero because the component of function normal to the common edge is unity.

An undesirable line integral will appear at the junction as shown in Figure 5 if the Gauss theorem is employed to transfer the differential operators $\nabla$ or $\nabla^{\prime}[17]$ just because the $\phi$ in the two triangles at the junction are not identical. 


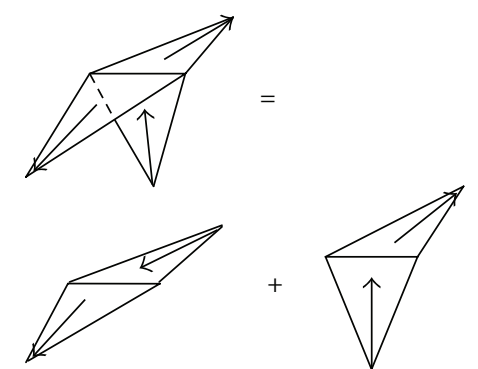

FIGURE 4: Division fashion of pair of triangles in this paper.

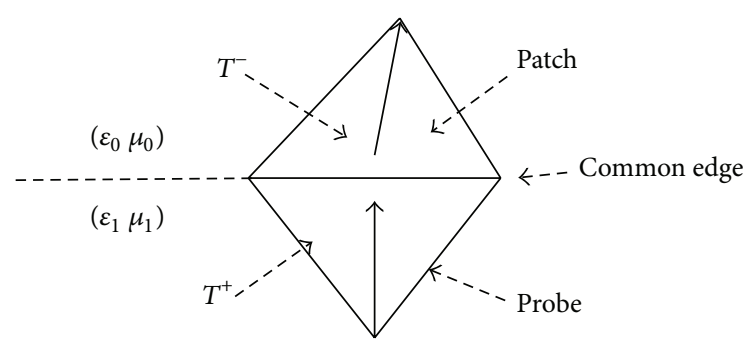

FIGURE 5: The triangle pair at the junction.

For example, when source point is located in the triangle pair at the patch and field point is located in the triangle pair at the junction,

$$
\begin{aligned}
\left\langle\nabla \phi, \mathbf{f}_{m}\right\rangle= & \int_{s} \nabla \cdot\left(\phi \mathbf{f}_{m}\right) d S-\int_{s} \phi \nabla \cdot \mathbf{f}_{m} d S \\
= & \oint_{l} \phi \mathbf{f}_{m} \cdot \widehat{\mathbf{n}} d l-\int_{s} \phi \nabla \cdot \mathbf{f}_{m} d S \\
= & \int_{l} \phi_{2} \mathbf{f}_{m}^{+} \cdot \widehat{\mathbf{n}}^{+} d l+\int_{l} \phi_{1} \mathbf{f}_{m}^{-} \cdot \widehat{\mathbf{n}}^{-} d l \\
& -\int_{T^{+}} \phi_{2} \nabla \cdot \mathbf{f}_{m}^{+} d S-\int_{T^{-}} \phi_{1} \nabla \cdot \mathbf{f}_{m}^{-} d S,
\end{aligned}
$$

where $l$ is the common edge as shown in Figure 5. $\phi_{2}$ is the electric scalar when source is located in the first layer and field is located in the second layer; and $\phi_{1}$ is the one when source locates in the first layer and field is located in the first layer.

The integration over the testing triangle surface and line is avoided through using the approximate Galerkin method, in which testing functions can be implemented at the centroid of the triangle and line. The surface integral over the source triangle is calculated using symmetric quadrature rules over the triangle.

Noticeably, calculating the self-element of impedance matrix at the junction is much complicated because four types of the dyadic Green's functions will be calculated, and difficult line integral singularity will also be met. The calculation formulation when both source and field triangle pairs position at the junction is as same as (21), but the meaning of $\phi_{2}$ and $\phi_{1}$ is not same as the ones of (21). $\phi_{2}$ is the electric scalar when field is located in the second layer, and $\phi_{1}$ is the one when field is located in the first layer. The

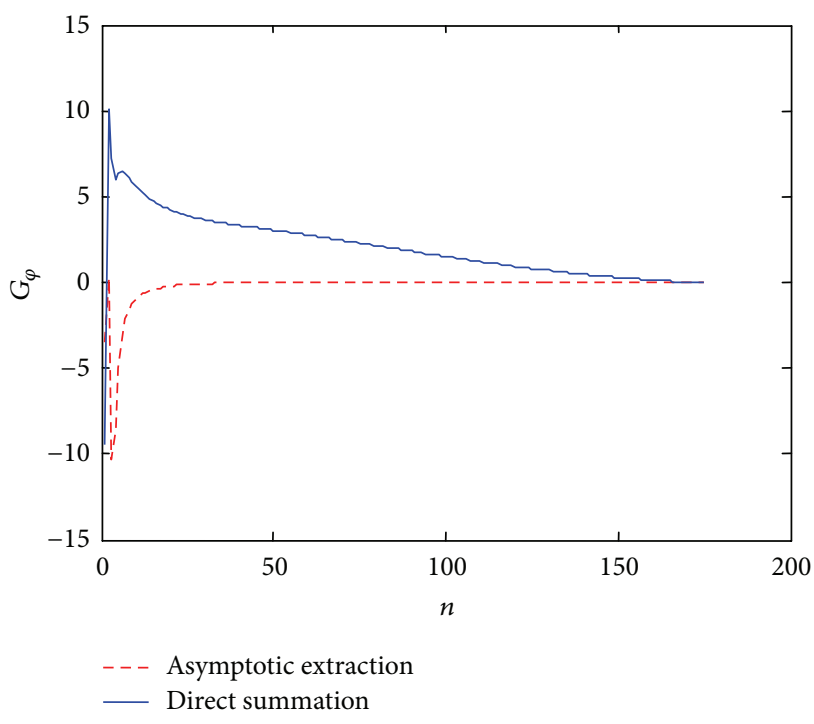

FIGURE 6: Convergence of $G_{\varphi}^{11}$ when source and field points are located in the same triangle at the patch.

position of source is in the first layer and second layer. In this case, the first term of (21) can be expressed as

$$
\begin{aligned}
\int_{l} \phi_{2} \mathbf{f}_{m}^{+} \cdot \widehat{\mathbf{n}}^{+} d l \\
=\int_{l} \int_{s^{\prime}} \nabla^{\prime} G_{\varphi} \cdot \mathbf{f}_{n} d S^{\prime} d l \\
=\int_{l} \int_{l^{\prime}} G_{\varphi}^{(22)} \mathbf{f}_{n}^{+} \cdot \widehat{\mathbf{n}}^{+} d l d l^{\prime}+\int_{l} \int_{l^{\prime}} G_{\varphi}^{(21)} \mathbf{f}_{n}^{-} \cdot \widehat{\mathbf{n}}^{-} d l d l^{\prime} \\
\quad-\int_{l} \int_{T^{+}} G_{\varphi}^{(22)} \nabla^{\prime} \cdot \mathbf{f}_{n}^{+} d S^{\prime} d l-\int_{l} \int_{T^{-}} G_{\varphi}^{(21)} \nabla^{\prime} \cdot \mathbf{f}_{n}^{-} d S^{\prime} d l .
\end{aligned}
$$

Then, (21) can be decomposed as 16 terms involving the calculation of four types of the dyadic Green's functions. Besides, the line integral singularity will be met when calculating (22):

$$
f=\int_{l} \int_{l^{\prime}} \frac{1}{\left|\mathbf{r}-\mathbf{r}^{\prime}\right|} d l d l^{\prime} .
$$

Hence, calculating the self-element of impedance matrix at the junction is much complicated. The patch is positioned at the interface between the two layers, and it can be regarded as being located at the bottom of free space or the top of the medium layer. Then, a smart method is regarding the two triangles at the junction as being located in the medium layer when calculating the self-element of impedance matrix at the junction.

\section{Calculated Results}

In this paper, the presented example in [13] has also been adopted, where the radius of the PEC spherical core is $5 \mathrm{~cm}$, with a spherical medium of $\varepsilon_{2}=2.47$ and a $0.32 \mathrm{~cm}$ thickness. 


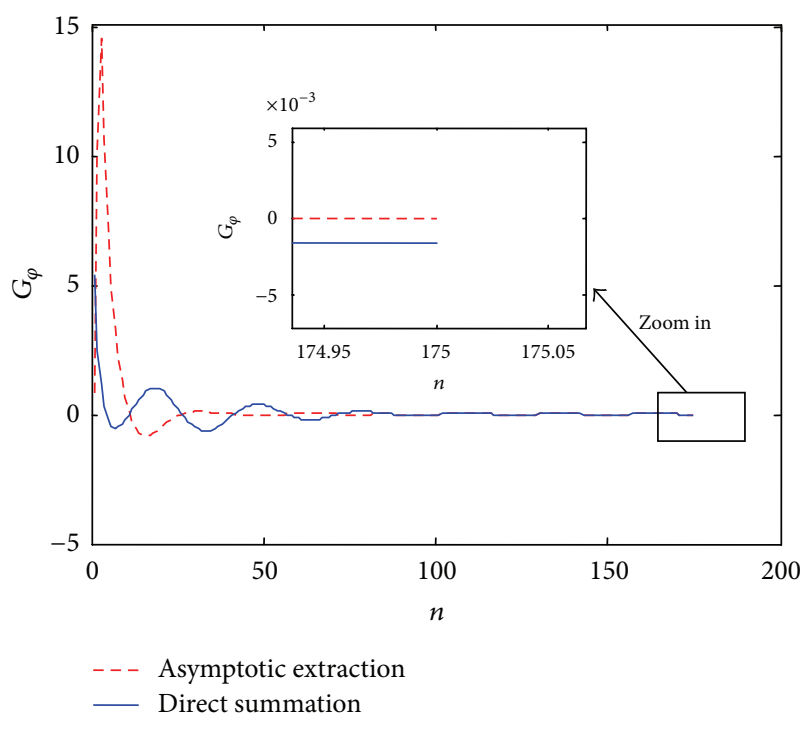

FIgURE 7: Convergence of $G_{\varphi}^{12}$ when source is located in the patch and field locate in the probe.

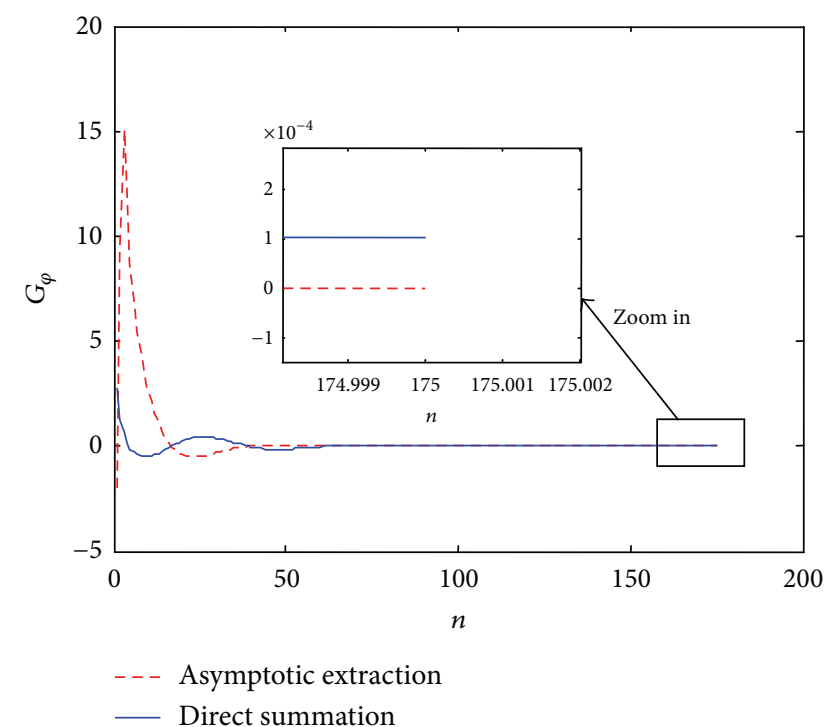

FIGURE 8: Convergence of $G_{\varphi}^{21}$ when source is located in the probe and field locate in the patch.

The conformal patch has an arc radius of $1.88 \mathrm{~cm}$, and the probe is located in an arc distance of $0.94 \mathrm{~cm}$ from the center of the patch. The width of the probe is $0.2 \mathrm{~cm}$. A delta gap voltage source has been placed at the base of the probe. The convergences of four types of Green's function are shown in Figures 6, 7, 8, and 9. The convergence has been accelerated significantly when the asymptotic extraction is applied in computing the summation between the source and field points that are located in the same layer and different layers.

The input impedance of the spherical microstrip antenna has been calculated compared with that cited in [13] as shown in Figure 10. The summation of $G_{\varphi}$ has been truncated

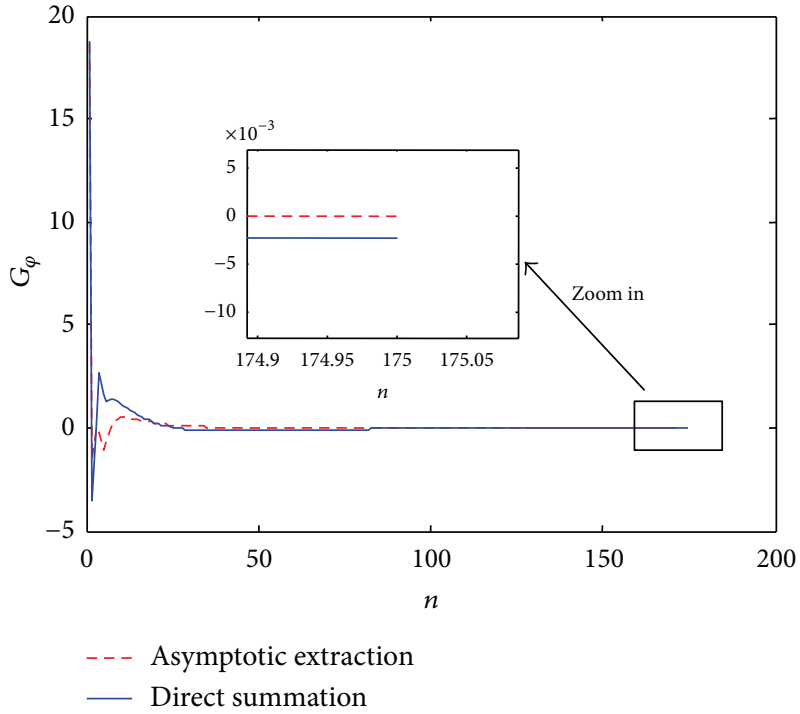

FIGURE 9: Convergence of $G_{\varphi}^{22}$ when source and field points are located in the same triangle at the probe.

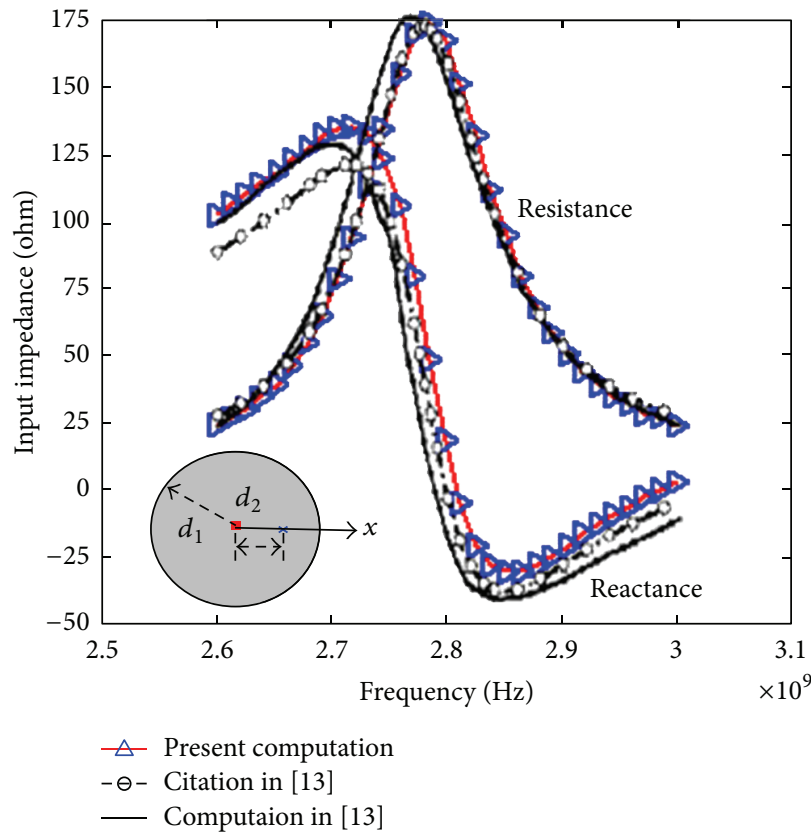

FIGURE 10: The comparison between the calculated result in this paper and the one of reference [13].

using 40 terms, which improves the computation efficiency significantly.

\section{Conclusions}

The input impedance of a three-layered spherical microstrip antenna has been calculated based on RWG triangular basis functions using mixed potential integral equation (MPIE). Asymptotic extraction approach is presented to accelerate the Green's functions convergence speed when source and 
field points are located in the same layer and different layers. A novel division fashion of pair of triangles is adopted to simplify impedance matrix element calculation at the junction of the probe and patch.

\section{Appendix}

When the source is located in the free space and the field is located in the medium, the asymptotic dyadic Green's functions of the $\overline{\mathbf{G}}_{e s}^{(21)}\left(\mathbf{r}, \mathbf{r}^{\prime}\right)$ are given as

$$
\begin{aligned}
\left.\overline{\mathbf{G}}_{e s}^{21}\right|_{a}= & \nabla \nabla^{\prime} G_{1} \\
= & \frac{j}{4 \pi k_{2}} \nabla \nabla^{\prime} \sum_{n=0}^{\infty}(2 n+1) P_{n}(\cos \gamma) \\
& \times\left\{w_{1} \frac{a_{2}}{r} j_{n}\left(k_{1} \frac{a_{2}^{2}}{r}\right) h_{n}^{(2)}\left(k_{1} r^{\prime}\right)\right. \\
\left.\overline{\mathbf{G}}_{e s}^{21}\right|_{a}= & \nabla \nabla^{\prime} G_{2} \\
= & \frac{j}{4 \pi k_{2} k_{1}} \nabla \nabla^{\prime}\left(w_{n} \frac{a_{2}}{r} \frac{e^{-j k_{2} R_{1}}}{R_{1}}+w_{2} \frac{e^{-j k_{2} R_{2}}}{R_{2}}\right),
\end{aligned}
$$

where

$$
\begin{aligned}
& w_{1}=\frac{\left(\left(\varepsilon_{2}-\varepsilon_{3}\right) /\left(\varepsilon_{2}+\varepsilon_{3}\right)\right)\left(\left(\left(\varepsilon_{1}-\varepsilon_{2}\right) /\left(\varepsilon_{1}+\varepsilon_{2}\right)\right)^{2}-1\right)}{2 \sqrt{\varepsilon_{1} \varepsilon_{2}} /\left(\varepsilon_{1}+\varepsilon_{2}\right)}, \\
& w_{2}=\frac{\left[1-\left(\left(\varepsilon_{1}-\varepsilon_{2}\right) /\left(\varepsilon_{1}+\varepsilon_{2}\right)\right)^{2}\right]}{2 \sqrt{\varepsilon_{1} \varepsilon_{2}} /\left(\varepsilon_{1}+\varepsilon_{2}\right)} \\
& R_{1}=\sqrt{r^{\prime 2}+\left(\frac{a_{2}^{2}}{r}\right)^{2}-2 r^{\prime}\left(\frac{a_{2}^{2}}{r}\right) \cos \gamma} \\
& R_{2}=\sqrt{r^{\prime 2}+r^{2}-2 r^{\prime} r \cos \gamma} .
\end{aligned}
$$

When the source is located in the medium and the field is located in free space, the asymptotic dyadic Green's functions of the $\overline{\mathbf{G}}_{e s}^{(12)}\left(\mathbf{r}, \mathbf{r}^{\prime}\right)$ are given as

$$
\begin{aligned}
& \left.\overline{\mathbf{G}}_{e s}^{12}\right|_{a}=\nabla \nabla^{\prime} G_{1}=\frac{j}{4 \pi k_{1}} \nabla \nabla^{\prime} \sum_{n=0}^{\infty}(2 n+1) P_{n}(\cos \gamma) \\
& \times\left\{w_{1} h_{n}^{(2)}\left(k_{2} r\right) j_{n}\left(k_{2} r^{\prime}\right)\right. \\
& \left.+w_{2}\left(\frac{a_{2}}{r}\right) j_{n}\left(k_{2} \frac{a_{2}^{2}}{r}\right) h_{n}^{(2)}\left(k_{2} r^{\prime}\right)\right\}, \\
& \left.\overline{\mathbf{G}}_{e s}^{12}\right|_{a}=\nabla \nabla^{\prime} G_{2}=\frac{j}{4 \pi k_{1} k_{2}} \nabla \nabla^{\prime}\left(w_{1} \frac{e^{-j k_{2} R_{1}}}{R_{1}}+w_{2} \frac{a_{2}}{r} \frac{e^{-j k_{2} R_{2}}}{R_{2}}\right) \text {, }
\end{aligned}
$$

where

$$
\begin{gathered}
w_{1}=\frac{2 \sqrt{\varepsilon_{1} \varepsilon_{2}}}{\varepsilon_{1}+\varepsilon_{2}}, \quad w_{2}=-\frac{2 \sqrt{\varepsilon_{1} \varepsilon_{2}}}{\varepsilon_{1}+\varepsilon_{2}} \frac{\varepsilon_{2}-\varepsilon_{3}}{\varepsilon_{2}+\varepsilon_{3}}, \\
R_{1}=\sqrt{r^{\prime 2}+r^{2}-2 r^{\prime} r \cos \gamma}, \\
R_{2}=\sqrt{r^{\prime 2}+\left(\frac{a_{2}^{2}}{r}\right)^{2}-2 r^{\prime}\left(\frac{a_{2}^{2}}{r}\right) \cos \gamma .}
\end{gathered}
$$

When the source and fields is located in the medium, the asymptotic dyadic Green's functions of the $\overline{\mathbf{G}}_{e s}^{(22)}\left(\mathbf{r}, \mathbf{r}^{\prime}\right)$ are given as

$$
\begin{gathered}
\left.\overline{\mathbf{G}}_{e s}^{22}\right|_{a}=\nabla \nabla^{\prime} G_{1}=\frac{j}{4 \pi k_{2}} \nabla \nabla^{\prime} \sum_{n=0}^{\infty}(2 n+1), \\
P_{n}(\cos \gamma) \times\left\{w_{1} \frac{a_{2}}{r} \times j_{n}\left(k_{2} \frac{a_{2}^{2}}{r}\right) h_{n}^{(2)}\left(k_{2} r^{\prime}\right)\right. \\
\left.+w_{2} \frac{a_{1}}{r} \times h_{n}^{(2)}\left(k_{2} \frac{a_{1}^{2}}{r}\right) j_{n}\left(k_{2} r^{\prime}\right)\right\}, \\
\left.\overline{\mathbf{G}}_{e s}^{22}\right|_{a}=\nabla \nabla^{\prime} G_{2} \\
=\frac{j}{4 \pi k_{2}^{2}} \nabla \nabla^{\prime}\left(w_{1} \frac{a_{2}}{r} \frac{e^{-j k_{2} R_{1}}}{R_{1}}+w_{2} \frac{a_{1}}{r} \frac{e^{-j k_{2} R_{2}}}{R_{2}}\right),
\end{gathered}
$$

where

$$
\begin{gathered}
w_{1}=-\frac{\varepsilon_{2}-\varepsilon_{3}}{\varepsilon_{2}+\varepsilon_{3}}, \quad w_{2}=\frac{\varepsilon_{1}-\varepsilon_{2}}{\varepsilon_{1}+\varepsilon_{2}}, \\
R_{1}=\sqrt{r^{\prime 2}+\left(\frac{a_{2}^{2}}{r}\right)^{2}-2 r^{\prime}\left(\frac{a_{2}^{2}}{r}\right) \cos \gamma}, \\
R_{2}=\sqrt{r^{\prime 2}+\left(\frac{a_{2}^{2}}{r}\right)^{2}-2 r^{\prime}\left(\frac{a_{2}^{2}}{r}\right) \cos \gamma .}
\end{gathered}
$$

\section{References}

[1] C. Y. Yin and M. Z. Hu, "An efficient analysis method for cylindrical ormal microstrip antenna fed by microstripline," International Journal of Antennas and Propagation, vol. 2012, Article ID 629748, 8 pages, 2012.

[2] K.-M. Luk and W.-Y. Tam, "Patch antennas on a spherical body," IEE Proceedings H, vol. 138, no. 1, pp. 103-108, 1991.

[3] R. Shavit, "Circular polarization microstrip antenna on a conical surface," IEEE Transactions on Antennas and Propagation, vol. 45, no. 7, pp. 1086-1092, 1997.

[4] B. Ke and A. A. Kishk, "Analysis of spherical circular microstrip antennas," IEE Proceedings H: Microwaves, Antennas and Propagation, vol. 138, no. 6, pp. 542-548, 1991.

[5] A. A. Kishk, "Analysis of spherical annular microstrip antennas," IEEE Transactions on Antennas and Propagation, vol. 41, no. 3, pp. 338-343, 1993. 
[6] A. C. de Castro Lima, J. R. Descardeci, and A. J. Giarola, "Circular microstrip antenna on a spherical surface," Microwave and Optical Technology Letters, vol. 5, no. 5, pp. 221-224, 1992.

[7] H.-T. Chen and Y.-T. Cheng, "Full-wave analysis of the annularring loaded spherical-circular microstrip antenna," IEEE Transactions on Antennas and Propagation, vol. 45, no. 11, pp. 15811583, 1997.

[8] Z. D. Dai and S. Lu, Dyadic Green's Functions in Electromagnetic Theory, Wuhan University Press, Wuhan, China, 2005.

[9] L.-W. Li, P.-S. Kooi, M.-S. Leong, and T.-S. Yeo, "Electromagnetic dyadic Green's function in spherically multilayered media," IEEE Transactions on Microwave Theory and Techniques, vol. 42, no. 12, pp. 2302-2310, 1994.

[10] S. K. Khamas, "Moment method analysis of an Archimedean spiral printed on a layered dielectric sphere," IEEE Transactions on Antennas and Propagation, vol. 56, no. 2, pp. 345-352, 2008.

[11] Z. Šipuš, P.-S. Kildal, R. Leijon, and M. Johansson, "An algorithm for calculating Green's functions of planar, circular cylindrical, and spherical multilayer substrates," Applied Computational Electromagnetics Society Journal, vol. 13, no. 3, pp. 243-254, 1998.

[12] Z. Sipus, S. Skokic, M. Bosiljevac, and N. Burum, "Study of mutual coupling between circular stacked-patch antennas on a sphere," IEEE Transactions on Antennas and Propagation, vol. 56, no. 7, pp. 1834-1844, 2008.

[13] S. K. Khamas, "Electromagnetic radiation by antennas of arbitrary shape in a layered spherical media," IEEE Transactions on Antennas and Propagation, vol. 57, no. 12, pp. 3827-3834, 2009.

[14] S. K. Khamas, "Asymptotic extraction approach for antennas in a multilayered spherical media," IEEE Transactions on Antennas and Propagation, vol. 58, no. 3, pp. 1003-1008, 2010.

[15] S. K. Khamas, "A generalized asymptotic extraction solution for antennas in multilayered spherical media," IEEE Transactions on Antennas and Propagation, vol. 58, no. 11, pp. 3743-3747, 2010.

[16] M. N. M. Yasin and S. K. Khamas, "Measurements and analysis of a probe-fed circularly polarized loop antenna printed on a layered dielectric sphere," IEEE Transactions on Antennas and Propagation, vol. 60, no. 4, pp. 2096-2100, 2012.

[17] K. A. Michalski and D. Zheng, "Electromagnetic scattering and radiation by surfaces of arbitrary shape in layered media-II: implementation and results for contiguous half-spaces," IEEE Transactions on Antennas and Propagation, vol. 38, no. 3, pp. 345-352, 1990. 

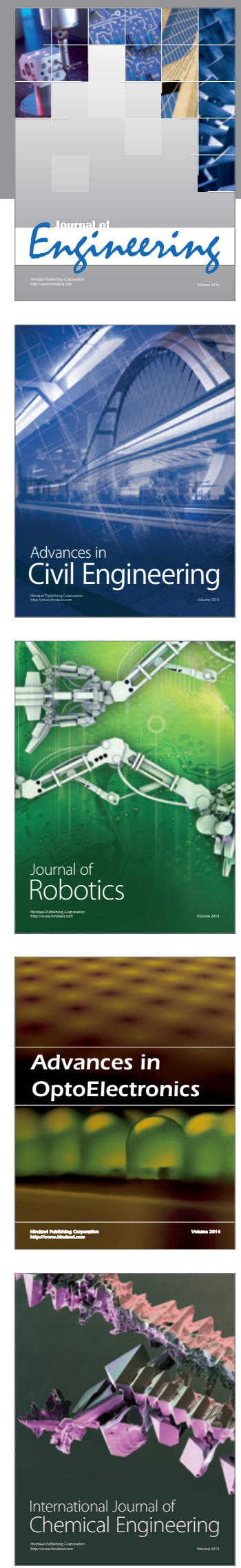

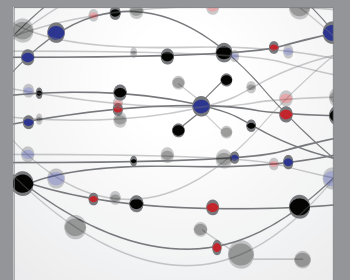

The Scientific World Journal
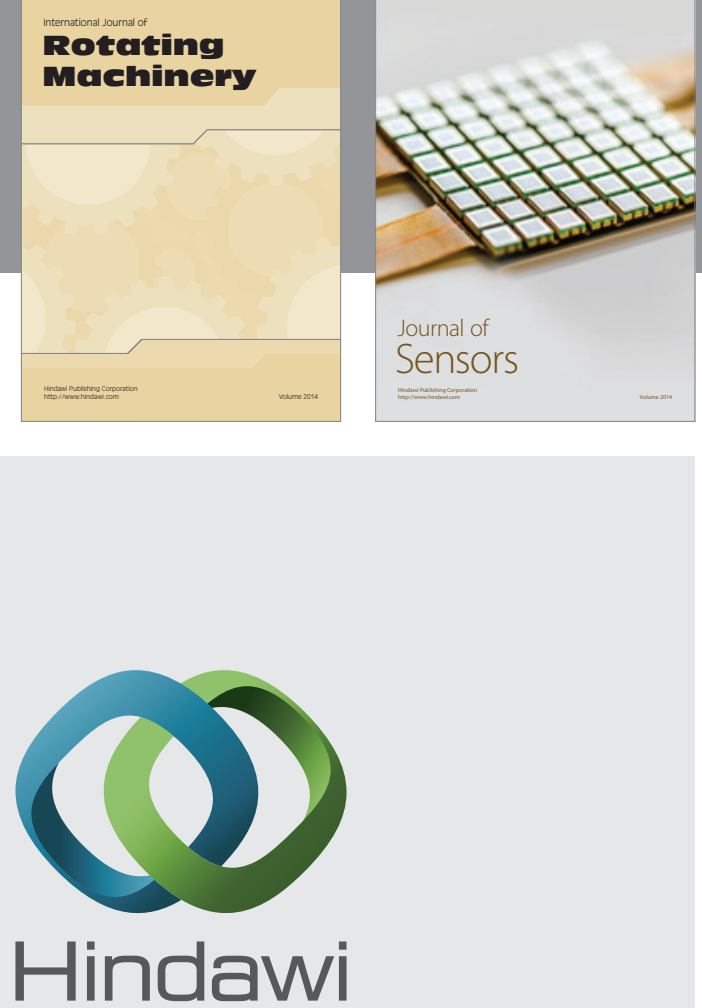

Submit your manuscripts at http://www.hindawi.com
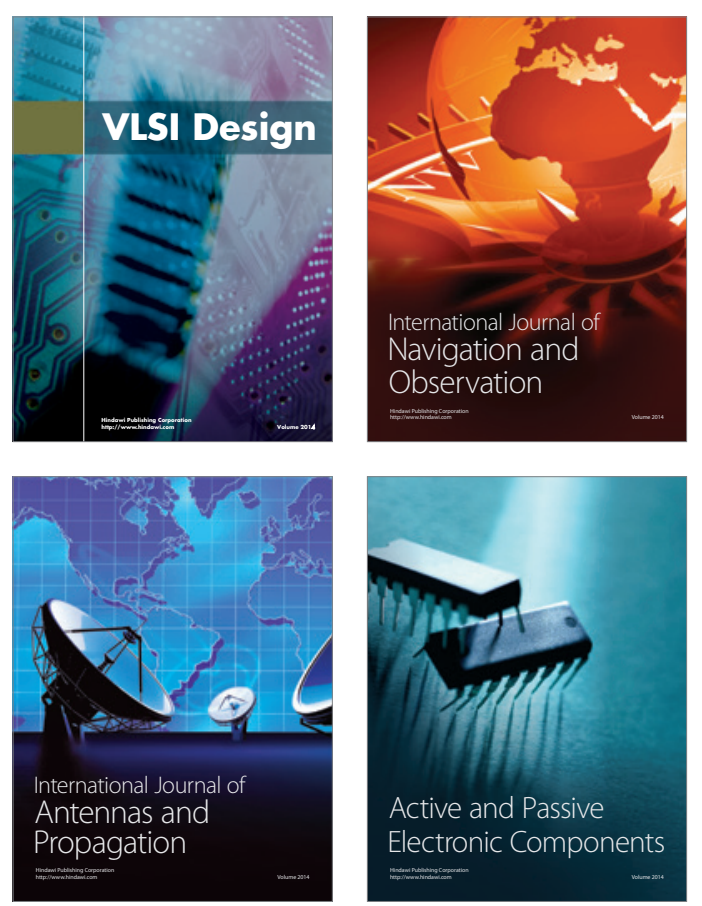
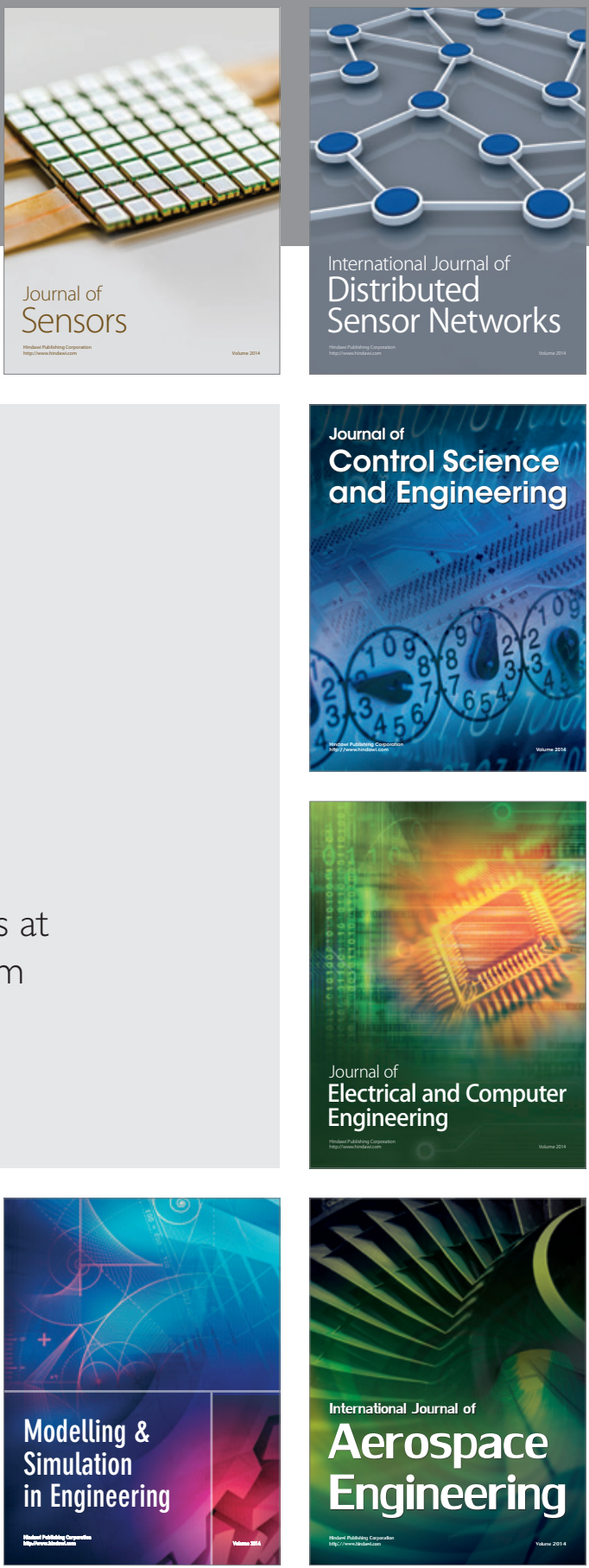

Journal of

Control Science

and Engineering
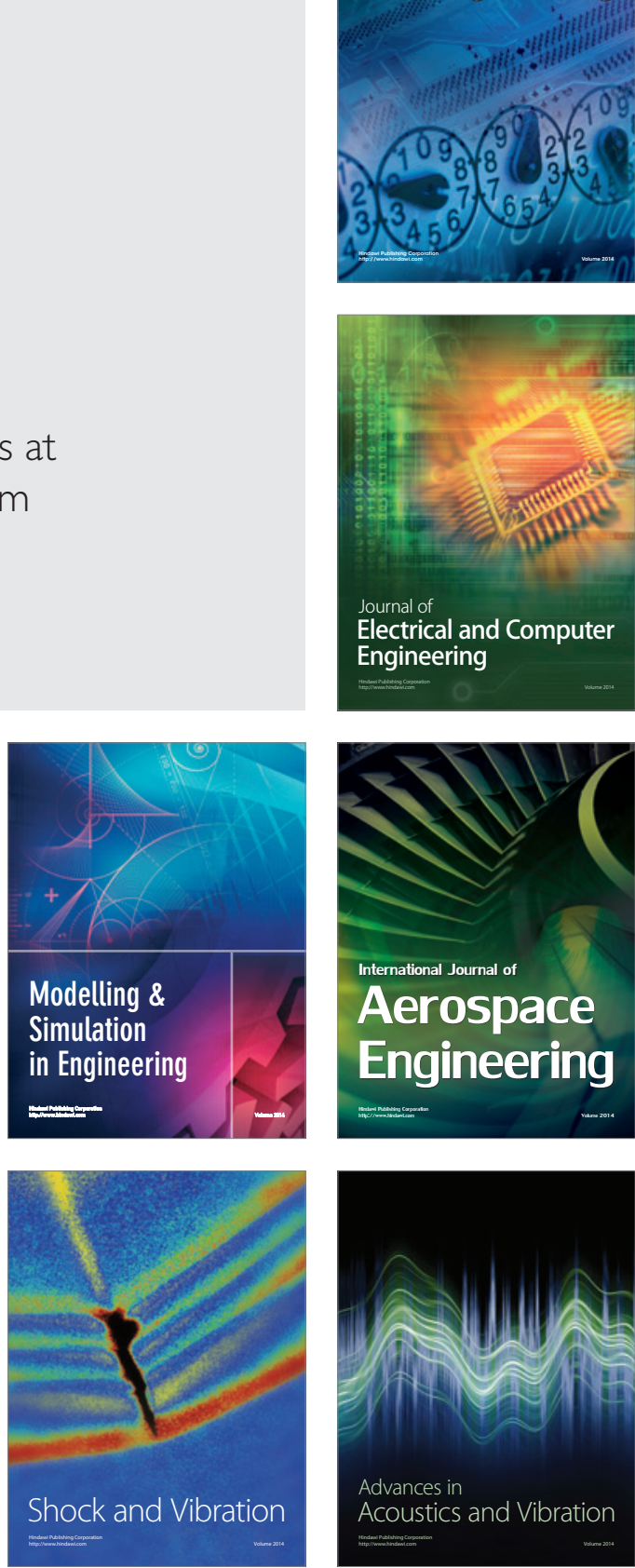